\title{
Demetra Christopoulou
}

\section{ON THE SYNTHETIC CONTENT OF IMPLICIT DEFINITIONS}

\begin{abstract}
This paper addresses the issue of stipulation in three cases of implicit definitions (postulates of scientific terms, systems of axioms and abstraction principles). It argues that the alleged implicit definitions do not have a purely stipulative status. Stipulation of the vehicles of the implicit definitions in question should end up with true postulates. However, those postulates should not be taken to be true only in virtue of stipulation since they have extra commitments. Horwich's worry emerges in all three kinds of implicit definitions under consideration, since the existence of meanings so that the alleged postulates are true depends on extra requirements that should be fulfilled. Moreover, if Ramseyfication method is applied to the three kinds of implicit definition, they are split up into two components from which the first one is broadly factual while the second one is purely stipulative. The paper argues that their definitional task in each case should be assigned to their second component i.e. their Carnap-conditional.
\end{abstract}

Keywords: implicit, definition, Ramseyfication, truth, a priori, synthetic, stipulation, conditional

\section{Introduction}

This paper addresses the issue of whether three kinds of implicit definition have a purely stipulative status. In section 2 , it argues that Horwich-type worries concerning stipulation in case of postulates of scientific terms emerge in cases of mathematical systems of axioms and abstraction principles too. In section 3, it applies Ramsey-Carnap method to the postulates in question to show their factual content that is captured by the Ramsey-sentence in each case. Thereby it defends the view 
that they should not be taken as true only in virtue of stipulation since they have extra commitments. In section 4 , it concludes that stipulation fails in all three cases and it suggests that the role of implicit definition should be assigned to their Carnap-conditionals.

\section{On truth obtained by stipulation}

According to a traditional account that connects implicit definitions with a priori truth, an implicit definition is a stipulation of a sentence that contains the definiendum as true. On this account, we take a sentence (or a range of sentences) that contains an unexplained expression (the definiendum) and other previously understood expressions and we stipulate it as true. In contrast with explicit definitions, the procedure does not aim to substitute the definiendum by a semantically equivalent expression. The aim of an implicit definition is to accrue a meaning to the definiendum of such a kind that a true proposition is indeed expressed by the sentence, just in virtue of the stipulation (cf. [5]). Hence, this account of stipulation is connected to the issue of a priori truth. If such an appropriate meaning is available then the effect of the implicit definition in question is that we acquire an a priori true proposition.

The recent discussion concerns implicit definitions of various kinds, especially postulates of scientific terms, mathematical systems of axioms and abstraction principles. In the first case, we may have a postulate of one or more theoretical scientific terms (e.g. 'electron'). As David Lewis puts it, a theoretical term is introduced by a certain theory $\mathrm{T}$ at a given stage in the history of science. We may take the postulate of a theoretical term to be a single sentence (if it was a set of sentences, we would take their conjunction) [9]. For example, if $f$ is phlogiston then its postulate $\mathrm{T}$ could be written down as an abbreviation "\# $f$ " ('\#' is a matrix that has to be read as 'such and such conditions' that $f$ should satisfy). A system of axioms is also regarded as an implicit definition of certain mathematical terms (e.g. 'line', 'point', 'number', 'zero', 'successor' etc.). IF MANY, the definienda obtain their meanings collectively on the basis of mutual relations they are taken to satisfy by a series of postulates. This idea of treating systems of axioms as implicitly defining certain mathematical terms comes from Hilbert (cf. $[12])$. The system of Euclidean axioms "\# $(f, g, h)$ " defines collectively the geometrical terms ' $f$ ', ' $g$ ', ' $h$ ' (: 'point', 'line', 'plane') by settling 
certain mutual relations that points, lines and planes satisfy. The third case is about abstraction principles. An account of abstraction principles as implicit definitions has been offered by Hale and Wright ([5] and [6]) in the context of Neofregeanism.

However, the process of stipulation faces well known difficulties. According to the traditional view stated before, the definiendum is to have the meaning it needs to have for the sentence in question (the vehicle of each implicit definition) to express a truth (cf. [5]). The process of stipulation has the effect of conveying a meaning for ' $f$ ' and that meaning contributes to the meaning of "\# $f$ ", so that this sentence comes to express the truth. We also assume, as Lewis [9] puts it, that the postulate (e.g. of a theoretical term like 'phlogiston') will be false in case the theoretical term in question is denotationless. ${ }^{1}$ Horwich has shown some serious difficulties. According to Horwich [7], when we assume that " $f$ ' should have the meaning it needs to have for "\#f" to be true, we presuppose that a. there is a meaning such that if ' $f$ ' has that meaning then "\# $f$ " is true and that b. there is at most one such meaning. Those points made by Horwich are known as the existence problem and the uniqueness problem respectively (cf. [7], [5]). Hence, if the appropriate meaning is available then "\# $f$ " gives a true proposition. But there may be no such meaning for ' $f$ ' or there may be more than one such meaning, Horwich objects. So, he believes that we have to reject the above traditional account of stipulation. Horwich suggests that it would be more precise to say that a meaning is accrued to the definiendum ' $f$ ' so that "\# $f$ " is held to be true or merely "\#f" is accepted. ${ }^{2}$

Let us recall Boghossian's suggestion how to deal with Horwich's critique. Boghossian [1] made a clarifying suggestion that the form of stipulation in question should rather be written as follows: "LET $f$ MEANING, if anything, what will make "\#f" true". This assertion can be true independently of whether or not there exists a meaning for ' $f$ ' so that it makes "\#f" true. Of course, to make this very assertion is far from claiming that "\# $f$ " is true. An extra premise is required (that ' $f$ '

1 He notes that this is a legitimate assumption for postulates although it does not hold for sentences in general.

2 Only the first objection of Horwich, that is the so called 'existence problem', will be taken under consideration in what follows. It is not the purpose of the paper to deal with the uniqueness problem and other problems Horwich indicated with concern to implicit definitions. 
does have the appropriate meaning) in order to infer that, in fact, "\#f" is true.

In similar words, the suggestion made by Boghossian is the following:

1. If the term ' $f$ ' is to mean what it does, then " $\# f$ " is true (for ' $f$ ' means whatever in fact makes "\#f" true). (Basic premise)

2. ' $f$ ' means what it does Therefore,

3. "\#f" is true. (Extra premise)

(Conclusion) [scheme I]

According to the scheme I, the truth of "\# $f$ " depends on both conditions 1 and 2. So, this postulate may not turn to be true (in case the alleged premises are not fulfilled). The moral that is drawn is that the very stipulation does not work if the assumptions 1 and 2 are not satisfied.

A part of the recent discussion was sketched above in order to focus the attention to a difficulty concerning the process of stipulation. The question that now arises here is whether the existence problem pointed out by Horwich with regard to definitions of scientific terms emerges also in case of systems of axioms and abstraction principles. The paper will argue that an analogous existence problem emerges when the availability of appropriate meanings for the definienda is concerned, so that the vehicles of those kinds of definitions express true propositions. I think that this point has been overlooked, since Horwich's worries have been discussed mainly with regard to definitions of scientific terms. This is due to certain worries that although stipulation of a sentence "\#f" as true (' $f$ ': a theoretical scientific term e.g. 'electron') should express a true thought, the proposition arising from such a stipulation may be empirically falsified. On the other hand, in case of mathematical implicit definitions, most people believe that stipulation works better since they give true propositions that cannot be empirically falsified. What the paper emphasizes is that though not empirically falsifiable, the alleged stipulations create a difficulty, similar to the one pointed out by Horwich. The effect of the process of stipulation is a proposition that should be true just in virtue of stipulation, but that truth depends on further semantic or metaphysical facts, which do not depend on us. 


\section{An analogous "existence problem" arising in case of axiomatic systems and abstraction principles}

As it has already been noted, systems of axioms are believed to be implicit definitions that define more than one terms (' $f$ ', ' $g$ ', ' $h$ ' etc.) simultaneously. Let "\# $(f, g, h)$ " stand for the system of Euclidean axioms, which lay down certain relations that the definienda ' $f$ ', ' $g$ ', ' $h$ ' ('point', 'line', 'plane') must satisfy. Horwich's WORRY can arise in this case too. According to the view which Horwich criticizes, we stipulate a sentence or a range of sentences "\# $(f, g, h)$ " as true. The definienda should acquire the meanings they need to have so that the postulates we get are true (in virtue of stipulation). However, there may be no appropriate meanings for ' $f$ ', ' $g$ ', ' $h$ ' so that the propositions expressed by the system in question are true. Of course, the main difference between a mathematical system of axioms and a postulate of a scientific term like 'electron' is that an empirical disconfirmation is not expected in the mathematical case. Yet, if only the very requirement of consistency is fulfilled, possible meanings are available for the definienda ' $f$ ', ' $g$ ', ' $h$ ' so that the postulates express true propositions. Hence, the appropriate meanings are available only with regard to the models of the system, not with regard to other interpretations that are not models. Moreover, as we will see later, the postulates in question have a broadly factual content. So the alleged truth, that we believed to hold just in virtue of stipulation, proves to depend on certain, independent on us, semantic requirements that should be fulfilled. Moreover, we will see that those requirements may concern metaphysical facts.

An abstraction principle

$(\mathrm{S}=) \quad(\forall F)\left((\forall G)\left[(\mathrm{S}(F)=\mathrm{S}(G) \leftrightarrow(F \approx G)] \quad\left(2^{\text {nd }}\right.\right.\right.$ order abstraction $)$

is taken to define implicitly an operator $\mathrm{S}$ (and, through it, the terms $\mathrm{S}(F), \mathrm{S}(G)$ formed by that operator). So, suppose ' $F$ ', ' $G$ ' are terms of a language which is already in use and ' $\approx$ ' is a relational predicate expressing an equivalence relation among $F$ and $G$. The already familiar language is extended by an operator $\mathrm{S}$ that applies to the already known expressions ' $F$ ', ' $G$ ' and produces new singular terms ' $\mathrm{S}(F)$ ', ' $\mathrm{S}(G)$ '. The bi-conditional settles truth conditions for the identity context " $\mathrm{S}(F)=$ $\mathrm{S}(G)$ ". An abstraction principle accrues meaning to the operator $\mathrm{S}$ and, through it, to the newly formed terms ' $\mathrm{S}(F)$ ', ' $\mathrm{S}(G)$ '. 
The bi-conditional of such a principle is stipulated as true (cf. [5], [6]), hence, the definiendum should acquire the meaning it needs to have so that the effect of the definitional procedure is a true proposition. Hence, the same question that cropped us in the previous cases of implicit definitions can arise again in this case (i.e. whether a meaning for the definiendum exists so that the very postulate in question becomes true). Nevertheless, in this case, abstraction's empirical disconfirmation is not expected, HOWEver, there may be no models. Hence, an extra requirement should be fulfilled. If the abstraction principle in question is satisfiable, then there are models of it and there will be meanings for the definiendum (the operator S). Yet, the existence of satisfiers does not immediately follow but only in those interpretations of the language that are models of the abstraction principle. The existence of appropriate meanings for the definienda depends on certain presuppositions that are independent on us, although not related to the empirical world. It is obvious that if the abstraction principle in question is not satisfiable, then there are no available meanings for the definienda and there are no semantic interpretations of the language in which the abstraction principle becomes true ${ }^{3}$. In addition, we have to take in account the fact that since an abstraction principle is not a logical truth, even if it is satisfiable, there will be certain interpretations (that are not models) in which it fails. Further we will see that abstraction principles convey a factual (but not empirical) content. Hence, the truth they express does not hold just by means of stipulation, but it depends on semantic and metaphysical facts.

I have to deal at this point with some remarks made by Hale and Wright concerning the conditional form of implicit definitions. Hale and Wright [5] have remarked that stipulations often lead up to arrogance. They call arrogant any stipulation of a sentence "\# $f$ " whose truth cannot be affirmed without a posteriori epistemic work [5]. They also have made alternative suggestions about how implicit definitions work well, but there is no space to discuss them here. I mention, however, that they believe the vehicle of implicit definition must be some sentence that its stipulation as true suffices to accrue meaning to the definiendum and that truth cannot be refuted by a posteriori evidence. What is at stake is that a stipulation of the truth of the sentence in question (the vehicle

3 According to Hale [6], if an abstraction principle does not meet the requirement of consistency, there is no meaning to be accrued to the definiendum, even if we suppose so. For example, Law $\mathrm{V}$ has been an inconsistent abstraction principle that Frege made use of in Grundesetze to introduce classes. 
of an implicit definition) is possible - that the truth of that sentence is indeed something which we can settle only in virtue of stipulation. They suggest that in order to avoid arrogance, definitions should be conditional in form. Stipulations given in the form of conditionals are not arrogant since they do not need a posteriori epistemic work to affirm the truth of the postulates in question.

Hence, Hale and Wright hold that abstraction principles in particular avoid arrogance because of their (double) conditional form. In the first place, it is correct to adopt the notion of arrogance Hale and Wright introduced. In fact, the conditional form of abstraction principles safeguards their resistance to any empirical disconfirmation so their epistemic a priori status is not at stake. However, this paper makes the further claim that the fact that abstraction principles do not need any a posteriori epistemic work is not adequate enough to confirm that their truth can be a matter of mere stipulation. Avoiding arrogance is not adequate enough to safeguard the stipulative status of abstraction principles. This paper questions the claim that the truth of an abstraction principle is the outcome just of a mere stipulation. Horwich's existence problem still arises in this case, since, as it was argued, the existence of such meanings that the very stipulation leads up to a true proposition depends on certain conditions that have to be met independently on our will. As we will see later those conditions concern semantic and metaphysical facts.

To corroborate my view that none of the three cases of implicit definitions has a purely stipulative status, I will attempt to point out that the relative postulates have a factual content. For this purpose, I need to apply a method that can distinguish between the factual and stipulative parts of each postulate in question.

\section{Ramseyfying implicit definitions}

Carnap elaborated a method how to distinguish between the analytic and the factual parts of a scientific theory; a method which Frank Ramsey himself had introduced too (Friedman [4]; Psillos [10], [11]). To cut a long story short, Carnap [3] held that a scientific theory $\mathrm{T}$ is logically equivalent to the conjunction ${ }^{\mathrm{R}} \mathrm{T} \&\left({ }^{\mathrm{R}} \mathrm{T} \rightarrow \mathrm{T}\right)$, where ${ }^{\mathrm{R}} \mathrm{T}$ is the Ramsey-sentence of the theory and ${ }^{\mathrm{R}} \mathrm{T} \rightarrow \mathrm{T}$ is the Carnap-conditional. The Ramseysentence ${ }^{\mathrm{R}} \mathrm{T}$ is an existential sentence we get if we replace the theoretical 
terms of the theory $\mathrm{T}$ by variables and then bind those variables with existential quantifiers. Then ${ }^{\mathrm{R}} \mathrm{T}$ says there are entities (or classes of) entities that REALISE the theory. Yet, a Ramsey-sentence never specifies the entities it asserts in a unique way, i.e. it does not describe a unique realisation of the theory. On the other hand, the Carnap-conditional ${ }^{\mathrm{R}} \mathrm{T} \rightarrow \mathrm{T}$ says that if there are entities that satisfy the Ramsey-sentence then those entities render the theory true. It implicitly defines the theoretical terms of the theory as satisfying certain conditions that are established by it. As Carnap put it, the conditional ${ }^{\mathrm{R}} \mathrm{T} \rightarrow \mathrm{T}$ is not a subject to either confirmation or disconfirmation and it is devoid of any factual content. Hence, it has the status of a meaning postulate.

If the Ramsey-Carnap method is applied to a postulate of a scientific term ' $f$ ' 4 then it can distinguish the factual content of it (captured by the Ramsey-sentence of it) from its analytic part (expressed by the relative Carnap-conditional). In this section, the Ramsey-Carnap method will be applied to all three cases of implicit definitions under investigation in order to show the synthetic status of the relative postulates and their extra commitments that block their ability to be purely stipulative. The point that will be emphasized is that mathematical postulates and abstraction principles convey an excess content too, captured by their Ramsey-sentences. Besides, their definitional role should be rather assigned to their Carnap-conditionals.

\subsection{Postulates of scientific terms}

The Carnap-Ramsey method will be applied to a postulate of a scientific term " $f$ '. Let us stipulate" $\# f$ " as true (e.g. "electrons satisfy such and such conditions").Such a meaning needs to be accrued to the term ' $f$ ' so that "\#f" expresses a truth. Further, the postulate "\# $f$ " that arises from such a procedure can be taken as equivalent to the conjunction " $\exists x(\# x) \&[\exists x(\# x) \rightarrow \# f]$ ". The effect of the definition should be that a true proposition is expressed (just in virtue of stipulation). However, the analysis indicates that the alleged truth requires the truth of the first component (the Ramsey-sentence), which says that there are entities that realize the initial postulate. The Ramsey-sentence captures the factual content of the postulate in question. Moreover, it may be

4 Lewis [9] applied the Carnap-Ramsey method only to postulates of scientific terms (the first kind) in his discussion of the stipulation issue with regard to questions about multiple or single realization of the alleged postulates. 
empirically falsified so the truth of the postulate depends on certain facts and it needs a posteriori work to be affirmed. Hence, the alleged truth depends on presuppositions independent on us that should be fulfilled and it is not just a matter of mere stipulation. This analysis shows why the alleged stipulation does not succeed in this case. On the other hand, the second component (the Carnap-conditional " $\exists x(\# x) \rightarrow \# f$ ") says that if there are entities that satisfy such and such conditions then $f$ satisfies those conditions. It accrues a meaning to the scientific term ' $f$ ' by denoting whatever (if anything) satisfies the conditions '\#'. Thereby it accomplishes the task of defining the initial definienda.

\subsection{Systems of axioms}

The second case concerns a system of axioms "\#(f,g,h)" e.g. the Euclidean system that defines implicitly the geometrical terms 'point', 'line', 'plane'. Again, those terms ' $f$ ', ' $g$ ', ' $h$ ' are to obtain such meanings that mutual relations hold among them. If the Ramseyfication method is applied in this case then the system of postulates can be divided into two components. The first is the existential Ramsey-sentence " $\exists x, y, z[\#(x, y, z)]$ " that replaces the theoretical terms ' $f$ ', ' $g$ ', ' $h$ ' by the variables $x, y, z$ and binds them by existential quantifiers. It says that there are (mathematical) entities that satisfy certain conditions \# laid down by the postulates. Hence, the postulates have a broadly factual content that is captured by the Ramsey-sentence. Of course this content is not empirical in this case, yet it is broadly factual, since it concerns existential commitments to a domain of abstract entities (e.g. mathematical entities). The system of postulates requires the truth of its Ramsey-sentence that depends on (independently on us) semantic and metaphysical facts. This analysis points out that the truth of the postulates is not a matter of mere stipulation but it depends also on certain semantic and metaphysical requirements. Besides, a general characteristic of the Ramsey-sentence is that it does not explicitly specify in any unique way the entities that it asserts. It allows for multiple semantic interpretations of them. ${ }^{5}$ The second component is the Carnap-conditional " $\exists x, y, z[\#(x, y, z)] \rightarrow \#(f, g, h)$ " which implicitly defines the theoretical terms ' $f$ ', ' $g$ ', ' $h$ ' by asserting that if there are entities that satisfy such

5 Multiple realization is a secondary issue here. What the paper aims to stress it that the Ramsey-sentence captures a factual content in the case of axiomatic systems too. 
and such conditions then $f, g, h$ satisfy those conditions. It is a meaning postulate and accomplishes the very task of the system to define ' $f$ ', ' $g$ ', ' $h$ ' (e.g. the initial definienda 'point', 'line', 'plane').

\subsection{Abstraction principles}

As for the third case, i.e. the case of abstraction principles, Ramsey's method will be applied here too, though this case is technically more complicated. For simplicity, let us consider Hume's principle ${ }^{6}$ :

$$
(\forall F)(\forall G)[(\mathrm{N}(F)=\mathrm{N}(G)) \leftrightarrow(F 1-1 G)]
$$

HP is a second order universally quantified bi-conditional, which says that for every $F$ and $G$ (concepts), the number of $F$ s is identical to the number of $G$ s if and only if $F$ and $G$ are 1-1 correlated. HP is quite important for the Neo-Fregean program, since the Peano axioms are derived by it in second order logic.

By Carnap-Ramsey's method, an abstraction principle can also be split up into two components, one of which is the Ramsey-sentence of it and the other is the relative Carnap-conditional.

The Ramsey-sentence of HP is a (higher order) existential sentence: "There is an operator S that works in such and such a way". It can be formulated as:

$$
" \exists \mathrm{S}(\# \mathrm{~S}) "
$$

(The matrix \# $:(\forall F)(\forall G)[\ldots(F)=\ldots(G) \leftrightarrow(F 1-1 G)])$

The second component is the Carnap-conditional that implicitly defines N (the Standard Numerical operator):

"If there is an operator S that works in such and such a way then the Standard Numerical operator N works this way":

$$
\text { " } \exists \mathrm{S}(\# S) \rightarrow \# \mathrm{~N} " \quad \text { (Quantification is over operators) }
$$

So, HP has a component with a broadly factual content (not empirical in this case), that is, a higher order existentially quantified sentence that

6 The argument of this subsection concerns abstraction principles in general. However, HP is an exemplary case of abstraction, because it has been taken to function legitimately as an implicit definition. This is due to the fact that it meets certain conditions (consistency, conservativeness, modesty, etc.) an abstraction principle has to meet in order to work successfully, according to Hale and Wright. 
asserts there is an operator $\mathrm{S}$ that works in a proper way. $\mathrm{S}$ is a secondlevel function which correlates concepts $F, G$ to their images $\mathrm{S}(F), \mathrm{S}(G)$ in such a way that $\mathrm{S}(F)$ is identical to $\mathrm{S}(G)$ if and only if $F$ and $G$ are 1-1 correlated. So abstraction principles convey an excess content too, that is captured by their Ramsey-sentences.

Further, this content is rich for the additional reason that the Ramsey-sentence does not uniquely specify the operator S in question, but it allows for multiple interpretations of it. Recall a remark made by Harrold Hodes [8], that there is no unique cardinality function that correlates concepts to their images (cardinal numbers) in the way HP exposes and that $\mathrm{N}$ (the standard Numerical Operator) is one of the many possible such functions.

In the previous section, I claimed that the truth of an abstraction principle is not something that holds just in virtue of mere stipulation, since the existence of appropriate meanings for the definiendum, such that the postulate in question expresses a true proposition, depends on certain, independent on us, conditions. Moreover, the Carnap-Ramsey method points out that the alleged truth of the postulate requires the truth of its first component, which conveys rich semantic and metaphysical commitments. So an abstraction principle is not purely stipulative. On the other hand, the second component (the Carnap-conditional) of the abstraction principle defines implicitly the initial definiendum, e.g. the operator $\mathrm{N}$, by saying that if there is an operator $\mathrm{S}$ working in the way indicated, then the Standard Numerical Operator N works exactly this way.

The point stressed in this paper is that stipulation as true fails in the three kinds of implicit definition under consideration. What I have been trying to do was to provide a unifying account of the function of three forms of implicit definitions and indicate that the truth the relative postulates are taken to express does not hold just in virtue of stipulation but it depends on further semantic and metaphysical requirements. The postulates in each case have a factual and a stipulative component and this characteristic shows their synthetic status ${ }^{7}$. In particular, in case of the first kind (postulates of scientific terms), the factual component has also an empirical content. The results that I have come across can now

7 Recall that Sellars ([13]) maintained that an explicit definition gives only analytic truths, however, if we search for a priori synthetic principles, then implicit definitions serve exactly this purpose. 


\begin{tabular}{|c|c|c|}
\hline Postulate & $\begin{array}{l}\text { Factual component } \\
\text { (Ramsey-sentence) }\end{array}$ & $\begin{array}{l}\text { Definitional component } \\
\text { (Carnap-conditional) }\end{array}$ \\
\hline "\#f" $(f=$ electron $)$ & " $\exists x(\# x) "$ & " $\exists x(\# x) \rightarrow \# f "$ \\
\hline $\begin{array}{l}\text { "\# }(f, g, h) " \\
\text { (Euclidean system } \\
\text { of axioms) }\end{array}$ & " $\exists x, y, z[\#(x, y, z)] "$ & $" \exists x, y, z \#(x, y, z) \rightarrow \#(f, g, h) "$ \\
\hline $\begin{array}{l}\text { Abstraction principle } \\
\text { e.g. HP }\end{array}$ & " $\exists S(\# S) "$ & $" \exists S(\# S) \rightarrow \# \mathrm{~N} "$ \\
\hline
\end{tabular}

Table 1.

be summarized in Table 1 by presenting (in different columns) the two components of the implicit definition in each case.

\section{A stipulative definition}

In the previous sections a view that implicit definitions of the three kinds under consideration are not purely stipulative was defended. However, each one has two components from the first of which is factual whereas the second one is stipulative. This second component (the Carnap-conditional) accomplishes the task of defining implicitly the initial definienda. The suggestion made here is that the definitional role of each one of the implicit definitions under consideration (postulates of scientific terms, mathematical systems of axioms and abstraction principles) should be assigned to their Carnap-conditionals. This assertion differs from Hale and Wright's suggestion that definitions should be conditional in form in order to avoid arrogance. It has been argued (in section 2) that a. avoiding arrogance does not safeguard any stipulative status and that $\mathrm{b}$. the fact that some postulates do not need a posteriori epistemic work is not adequate enough to confirm that their truth is a matter of mere stipulation. Even in case the initial implicit definition in question already has a conditional form (e.g. an abstraction principle) their Carnap-conditional should accomplish the definitional role instead of them. This is not merely due to the fact that the Carnap-conditionals avoid (epistemic) arrogance, but that they convey no factual content at all. The point that is stressed here is that we need to find such a 
formulation of the vehicle of implicit definition that excludes any factual content from the candidate postulate. A Carnap-conditional has a purely stipulative character that is due to some special characteristics that are described below. Recall that Carnap himself characterized those conditionals as (analytic) meaning postulates.

I. The way stipulation has to work is to assume that we let the definienda mean, if anything, whatever will make our postulates true. A Carnapconditional has such a form that not only prevents any empirical disconfirmation, but it safeguards that it is factually empty as well. Let stipulate e.g. " $\exists x(\# x) \rightarrow \# f$ " as true. The postulate in question has a Ramsey-sentence that is trivially true, hence it is devoid of any factual content (for this assertion, also cf. Psillos [11]). This characteristic of factual emptiness makes an important difference between a Carnapconditional and any other kind of implicit definition.

II. A second characteristic related to the one described previously is that Carnap-conditionals can be regarded as unconditionally assertable principles in the sense that Sellars has indicated in his [13]. Sellars characterizes as unconditionally assertable those principles which are neither empirical generalizations nor deduced by other principles. Those are regarded as constituting certain conceptual frameworks. Hence, they define implicitly certain theoretical terms, they are implicit definitions. It is true that Carnap-conditionals are not derived either by deduction or by induction. After stipulation, the Carnap-conditionals do not make any factual claims and we can unconditionally accept them.

III. Carnap-conditionals state a choice of names that are given to a sequence of values of the existential variables that occur in the antecedent of the conditional. They assign meanings to a range of theoretical terms by laying down the assertion that the definienda should acquire meanings according to certain conditions established by the Ramsey-sentence. Accordingly, Carnap-conditionals have the proper formulation in order to work as stipulations. In particular, on the base of section 3, the Carnapconditional of an implicit definition (of the three kinds investigated) says that if there are any items that satisfy such and such conditions (established by the relative Ramsey-sentence) then the (initial) definienda should denote them. According to the characteristics above, Carnapconditionals have a purely stipulative status. Hence, the definitional role of the alleged implicit definitions (of the three kinds investigated so far) should be assigned to their Carnap-conditionals. 
Acknowledgments. This research has been co-financed by the European Union (European Social Fund - ESF) and Greek national funds through the Operational Program "Education and Lifelong Learning" of the National Strategic Reference Framework (NSRF) - Research Funding Program: THALIS-UOA-Aspects and Prospects of Realism in the Philosophy of Science and Mathematics (APRePoSMa).

\section{References}

[1] Boghossian, P., "Analyticity reconsidered", Noûs 30, 3 (1996): 360-391.

[2] Carnap, R., "Meaning postulates", Philosophical Studies III, 5 (1952): 65-73.

[3] Carnap, R., "Observation language and theoretical language" (1958), in: J. Hintikka (ed.), Rudolf Carnap, Logical Empiricist, Dordrecht, Holl., D. Reidel Publishing Company, 1975.

[4] Friedman, M., "Carnap on theoretical terms: structuralism without metaphysics", Synthese 180 (2011): 249-263.

[5] Hale, B., and C. Wright, The Reason's Proper Study, Oxford, Clarendon Press, 2001.

[6] Hale, B., "A response to Potter and Smiley: abstraction by recarving", Proceedings of the Aristotelian Society 101, 3 (2001): 339-358.

[7] Horwich, P., Meaning, New York, Oxford University Press, 1998.

[8] Hodes, H., "Logicism and the ontological commitments of arithmetic", Journal of Philosophy, 81 (1984): 126-135.

[9] Lewis, D., "How to define theoretical terms", Journal of Philosophy 13 (1970): 427-446.

[10] Psillos, S., Scientific Realism, London \& New York, Routledge, 1999.

[11] Psillos, S., "Ramsey's Ramsey-sentences", pages 67-90 in Cambridge and Vienna: Frank P. Ramsey and the Vienna Circle, M. C. Galavotti (ed.), London, Springer-Verlag, 2006.

[12] Resnik, D., "The Frege-Hilbert controversy", Philosophy and Phenomenological Research 34 (1974): 386-403.

[13] Sellars, W., "Is there a synthetic a priori?", Philosophy of Science 20 (1953): 121-138.

Demetra Christopoulou

University of Athens,

Department of Philosophy and History of Science 117 Plastira st.

GR 17122, Athens, Greece

chrdemet@yahoo.gr 